\title{
Commentary
}

\section{Architectural thinking and some aspects of technical creativity}

\author{
G. MANOLESCU \\ Inst. Central pentru Conducere si Informatica, B-dul Miciurin \\ 8-10, Sector 1, Bucharest, Romania
}

We are largely surrounded by and included in increasingly complex technical systems. These systems often border on the absurd even though they rely on logical and physical forms. This is because they move away from the logical 'simplicity' of natural forms. This paper approaches some specific aspects of technical products, i.e. those connected to their formative structure. M. Draganescu emphasized that the aesthetics and the functions of a product are determined by the consonance between the formative structure and the natural structure, and called this 'architectural thinking'. As an example, consider the well-known 'golden number' found both in natural crystals and in artistic and technical products such as the Parthenon.

Two questions which this paper addresses are: What is a formative structure? How can the consonance, or harmony, between such a structure and that of a 'natural' one be approached?

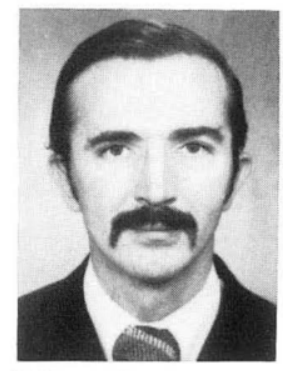

Gorun Manolescu has a Ph. D. in Engineering Science and graduated at the Oil and Gas Institute, Bucharest, Romania in 1960.

During 1977-1979 he was professor of information systems at the Oil and Gas Institute. He is now senior researcher at the Central Institute for Management and Informatics, Bucharest, Romania, working in the area of CAD. Dr. Manolescu has published more than 50 articles, books and research papers in the areas of informatics, management, automata and technical creativity. $\mathrm{He}$ has conducted many large-scale information systems projects, e.g., the technical-scientific information system of the Oil and Gas Industry (Romania), CAD systems in the fields of mechanical engineering, civil engineering, etc. (Romania).

North-Holland

Human Systems Management 4 (1984) 226-228
The twentieth century ushered in an unprecedented investigation of architectural thinking. Analytical modes of thought were introduced, as well as a thirst for understanding and harmony between and among natural and man-made structures. Ways to cross disciplines were proposed, by explicating such concepts as 'interdisciplinary', 'plura-disciplinary,' and 'transdisciplinary'. Systematic, cybernetic, semiotical, and communicational approaches resemble most closely the transdisciplinary approach and appear to be most realistic.

What makes architectural thinking and an architectural approach differ from other modes of transdisciplinary thought in their constructive characters? This mode of thinking transcends others, in that it has the main advantage of genetic epistemology: maintaining that the value of the human contribution is a unique and efficient means of accomplishing a goal. Architectural thinking aims to develop the human capacity for efficient activity in the environment, in opposition to the old ideal of static, contemplative knowledge.

The architectural 'thinking/approaching' model tries to cover in every possible way all the functions of a system, including the technical, human and aesthetic aspects. For this reason it is not subsumed in the system but instead exceeds the system. Of special interest is the way the integration is viewed within the framework of architectural thinking. In opposition to the systemic 'objective' gestalt, the architectural gestalt cannot be separated from the subject due to the fact that it is the determining factor that creates and intercepts that 'whole' of the architecture and produces a 'unique, scientifically undescribable state'.

The theme of this paper is that the architectural gestalt is far from an uncontrolled and uncontrollable subjectivism - and the sphere of technical 
creativity has been chosen to serve as an example.

We shall start from the observation that imagining the architecture of a future technical product implies imagining a structure that is to realize it. But obviously the architecture must not and cannot be reduced to a structure since the system cannot be reduced to its structure. If we still insist upon only the structural aspect of the architecture of a future technical product that is because, as a first approach, we consider it sufficient to tackle features of the architectural gestalt.

From the designer's point of view, the product can be seen as a hierarchy of structures. Such a hierarchy may be the following:

(a) A first structure, directly visible, of the 'physical' product-object, which we call the physical structure.

(b) The second structure, more profound, is the logical structure of the functions of the product, which determine and condition the physical structure, or, in other words, which are implemented by means of a physical structure.

(c) The third structure, the most profound, which we call the formative structure, determines and conditions the logical structure or, in other words, is implemented by means of the logical structure. ${ }^{1}$

As far as we know, the formative structure of the technical product has not yet been explicitly emphasized. This structure is to a great extent responsible for the appearance (or absence) of the architectural gestalt. In the designer or the user of a technical product, architectural gestalt produces a state similar to the one called 'job satisfaction' in Anglo-Saxon literature. There are several reasons to dwell at length upon this kind of structure.

The first reason emphasizes the intimate correspondence among the three kinds of structures (physical, logical, and formative). It is about a certain class of technical products, namely those meant to harmoniously reproduce sounds. Indeed it seems that there exists here a perfect joining between certain simple numerical ratios (for example, between the thickness and the length of strings in a string instrument), product functions, and the

\footnotetext{
1 The term 'implementation' emphasizes the fact that between the logical structure and the physical structure there appears an implicit interconditioning (from physical to logical) by some constraints imposed by the practical at a given time within existing possibilities of realization.
}

structure that gives shape to the physical object when it is manufactured. A second reason emphasizes the harmony between the efficiency and aesthetics of the 'aerodynamic form', and highlights the natural character of this form found in birds, on an industrial scale. ${ }^{2}$

Eventually, we have a related example of the 'computer architecture' field (a term circulating among the specialists in informatics). Described here is the designing of a profound structure, known as 'top-down-hierarchical structure', through which the functions of the product are ordered. Such a structure is often found in nature. It is similar to the structure of 'natural' reasoning. Clarity and efficiency of design are the ideals of the 'hierarchical top-down structure'.

What perspective can we gain on formative structures from this discussion? What defining characteristics of a formative structure could be put into relief from our discussion?

First of all, if we are to look closely at the outlook of the third kind of structure, we notice that this formative structure has a maximum of potentiality in comparison with a respective logical physical structure. Actually, it implies the existence of an infinity of logical structures determining updating, as well as a logical structure involving the existence of a new infinity that can be updated.

Secondly, one notices a kind of 'a priorism' of a formative structure that could be linked to some abstract structures of the human thought process.

Finally, one notices that the efficiency of a technical product appears when its formative, pro* found structure has a natural character. It seems that 'naturalness' of the formative structure is given by its consonance with that of a structure already created by nature (for example, the 'golden number' described earlier).

But how can the consonance between the for-

\footnotetext{
${ }^{2}$ It is a known 'mathematical' way of determining the ratio between lengths of homogeneous strings and of strings of the same thickness, meant to reproduce the sounds of the scale. It derives out of the Pythagorean principle, according to which numbers are the 'model of every thing'. It is also worth mentioning that at the end of the 11th century a monk called Theophile established a strictly empirical rule for manufacturing the bells that ring the key note, the third, the quint, and the octave. According to him, the bells must have their diameters proportioned to $30,24,20$, and 15 , and their weights in inverse ratio to $80,41,24$, and 10 .
} 
mative structure of a future technical product and the appropriate structure already existing in nature be realized? The importance of defining the right answer to this question is obvious. In fact, we consider that this consonance has the power to determine the appearance of the architectural gestalt of the future product. We hope that we are correct in considering that the problem of a proposed solution of consonance marks the limits (aesthetics) of the industrial design field. In this instance we are tempted to assimilate the architectural gestalt's elaboration to a process of an intuitive nature. Further development of such processes, after some 'incubation' period which is marked by fixating sometimes intense concerns on the subject, spontaneously reveals the solution, assuming the form of 'insight'. Such a solution immediately reveals itself (as a kind of direct mental 'perception') and it thoroughly and conspicuously imposes itself upon the subject. At this stage of such processes, it is necessary for the principle of minimization (efficiency) and simplicity to be revealed.

The value of a technical product is given both by its utility and its performance. If taken broadly, utility is seen with respect to the period of product elaboration (by identification in detail with functions). Performance, especially in the case of large-scale systems, is often almost completely ignored. On the contrary, we think that only the consciousness of performances (in terms of qualitative and/or quantitative ones) is the catalytic factor which encourages and brings about, during the technical creativity process, the appearance of adequate formative structures from the point of view shared by us (consonance). Further discussion on this subject is useful considering our original premise: that we are largely surrounded by and included in increasingly complex technical systems, which move away from the simplicity of the 'natural' forms.

The ideas of architectural gestalt and architectural thinking as approaches to design significantly updates the problem of intercorrelation and interdependence of the subject to/on the object. No doubt, there is a disturbing subjectivism, which can be an obstacle to acquiring knowledge. But there exists a creative and constructive subjectivism which weaves the threads through the true knowledge and activity out of respect to the objective and natural truth. All the effort is made with a view toward creating a code appropriately reflecting the object, which will broadly help to discover an adequate solution reflecting the laws of the object on the strength of its cognition, alteration, or manufacture. 